\title{
Variables affecting the probability of complete fusion of the medial clavicular epiphysis
}

\author{
Lynn Meijerman • George J. R. Maat • \\ Ronald Schulz • Andreas Schmeling
}

Received: 19 March 2007 / Accepted: 19 June 2007 / Published online: 2 October 2007

(C) Springer-Verlag 2007

\begin{abstract}
In this study, we have combined data on clavicle fusion from different studies and applied a binomial logistic regression analysis. As such, we aimed to assess whether or not variables such as sex, socioeconomic status, and ethnicity influence the probability of having mature, i.e., completely fused clavicles at a given age. We further explored whether the method of clavicle examination, i.e., diagnosis from either a dry bone specimen, an examination of X-rays, or an examination of computed tomography scans, affects the probability of being diagnosed with mature clavicles. It appeared that only ethnicity did not significantly affect this probability. Finally, we illustrated how the logit model may be used to predict the probability of being diagnosed with mature clavicles.
\end{abstract}

Keywords Age estimation - Clavicle - Medial epiphysis · Epiphyseal fusion

\section{Introduction}

The medial (or sternal) clavicular epiphysis matures relatively slowly. In the human skeleton, it is generally the last

\section{Meijerman $(\bowtie)$}

Netherlands Forensic Institute,

P.O. Box 24044, 2490 AA The Hague, The Netherlands

e-mail: L.meijerman@nfi.minjus.nl

G. J. R. Maat

Barge's Anthropologica, Leiden University Medical Center,

P.O. Box 9600, 2300 RC Leiden, The Netherlands

R. Schulz $\cdot$ A. Schmeling

Institute of Legal Medicine, University of Münster,

Roentgenstraße 23,

48147 Münster, Germany long bone epiphysis to fuse. Its developmental stage is therefore useful when estimating age at death in the post-pubertal period [1] or the age of living persons involved in criminal proceedings $[13,15]$. In the Netherlands, a radiological clavicle examination may be applied to verify the age of young asylum seekers without valid identification documents.

Various authors have studied clavicle development to determine the age interval in which fusion of the clavicular epiphyses occurs. Most of the published studies were based on the examination of dry bone specimens $[3,6,9,10,17$, 18]. When dealing with living individuals, examiners analyzed either X-rays [4, 13] or computed tomography (CT) scans $[5,15,16]$. In a number of studies, the results for different ethnic groups were evaluated or the results for males were compared with those for females. In most studies, such comparisons were, however, hampered by a relatively small sample size.

In this study, we aim to assess from a large sample whether or not variables such as sex, socioeconomic status, and ethnicity affect the probability of having completely fused clavicles at a certain age. We further aim to compare results from X-ray and CT scan examinations with those from studies based on dry bone specimens. To do so, we have combined data from different studies and performed a binomial logistic regression analysis. We then illustrate how the resulting model can be applied to estimate the probability of completely fused clavicles at a given age.

\section{Data}

Published and unpublished data on the individual subjects, taken from various studies on clavicle development [3-6, 8, $10,13,15,18]$ were combined. For each individual, information on the calendar age in years was combined with 
data on the fusion status of the clavicle, i.e., exhibiting a complete bony fusion of epiphysis and diaphysis (i.e., mature) or not. When data for both left and right clavicles were published, we used the side for which most records were provided. Data on the sex and ethnicity of the individuals were copied when available. In the context of this study, we defined ethnicity as being either of European (Caucasian), Asian, or Afro-American descent. It was further recorded which method was used for determining the fusion status, i.e., using dry bone specimens, X-rays, or CT scans.

The socioeconomic status of individuals was estimated from the human development index (HDI) associated with the country in which the study took place. The HDI is a summary composite index that measures a country's average achievements in three basic aspects of human development: longevity, knowledge, and the standard of living [19]. The HDI is used by the United Nations Development Programme, and data are available from 1975 onward. To accommodate data from two American studies that took place before this year $[6,18]$, we extrapolated the HDI by first calculating the linear regression of the HDI on time.

In total, data of 3,552 individuals aged 15-33 years were combined. Figure 1 shows the age distribution within the sample. Table 1 provides further information on the number of individuals per category.

\section{Statistical analysis}

Analysis of the data was performed using the SPSS (version 10.0) software package for statistical analysis. We carried out a binomial logistic regression analysis to assess the effect of various variables on the probability of mature clavicles. In logistic regression, the dependent variable is transformed into a logit variable, i.e., the natural $\log$ of the odds of the dependent occurring or not. This transformation ensures that the estimated probabilities are between 0 and 1. A logit model is a form of the generalized linear model.

Age, sex, ethnicity, and socioeconomic status were considered as potential predictor variables for the probabil-

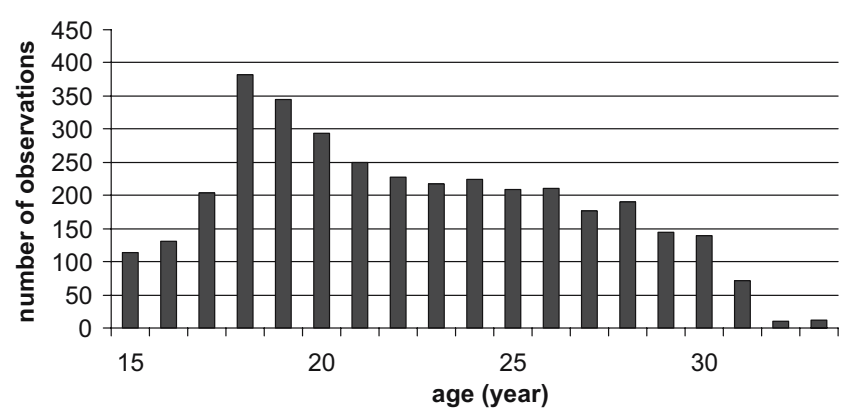

Fig. 1 Age distribution of individuals in the sample
Table 1 Number of individuals per category

\begin{tabular}{ll}
\hline Category & Values \\
\hline Total number of individuals & 3,552 \\
Sex & \\
Males & 2,133 \\
Females & 1,123 \\
Unspecified & 296 \\
Ethnicity & \\
European descent & b \\
Afro-American descent & 1,316 \\
Asian descent & 287 \\
Unspecified & 695 \\
Method & 1,254 \\
Dry bone specimen & \\
X-ray & 1,374 \\
CT scan & 1,326 \\
\end{tabular}

${ }^{a}$ A small number of individuals in McKern and Stewart [6] were of Afro-American descent. As no individual data were provided, all 374 individuals are classified "of European descent".

b "Of European descent" includes Caucasian and Latino individuals from North America.

ity of mature clavicles. The method of clavicle examination was further considered as a factor influencing the probability of being diagnosed with mature clavicles. Age and socioeconomic status (i.e., HDI) were analyzed as covariates. Sex, ethnicity, and the method of clavicle examination were analyzed as fixed factors. Possible interaction between these fixed factors and the covariate age was also investigated. The initial (exploratory) logit model may be described by the following equation:

$$
\begin{aligned}
& \ln [p /(1-p)]=\alpha+\beta_{\text {age }} \times \text { age }+\beta_{\mathrm{HDI}}{ }^{\times} \mathrm{HDI}+\gamma_{\text {sex }}+\gamma_{\text {ethnicity }} \\
&+\gamma_{\text {method }}+\beta_{\text {sex } \times \text { age }} \times \text { age }+\beta_{\text {ethnicity } \times \text { age }} \times \text { age } \\
&+\beta_{\text {method }} \times \text { age } \\
& \times
\end{aligned}
$$

where $p$ denotes the probability of the dependent, $\alpha$ the intercept, $\beta$ the estimated effect of a covariate or factor-bycovariate interaction term on the logit of the probability, and $\gamma$ the estimated effect of a fixed factor.

Variables to be kept in the model were selected by means of backward elimination using the Wald test. Insignificant interaction terms were successively dropped. After each alteration to the exploratory model, possible significance of the remaining variables was reassessed. A $p$ value lower than 0.05 was considered to be statistically significant.

Not all studies that were used as a source for our data provided individual data on sex or ethnicity. Consequently, the described model (M1) could be tested on the data of a subgroup of 2,002 individuals only. No data from CT scans were available for this subgroup. To be able to include data from all available studies, we therefore also tested simpli- 
fied models. For the first of these simplified models (M2), we omitted the variable ethnicity and the associated interaction term from the initial model. This model could be fitted to the data of a subgroup of 3,256 individuals. For this subgroup, data resulting from the evaluation of CT scans were drawn from a single study [15], as the second available study [5] did not provide details on the sex of individuals. As a next step, we therefore further omitted the variable sex and the associated interaction term from the initial model. The resulting model (M3) could be fitted to the data of all 3,552 individuals. As a final step, we assessed the significance of the change in $-2 \log$ likelihood between the resulting model and a nested model including the main terms only. We further compare the Nagelkerke pseudo $R^{2}$. As such, we compare the difference in model fit.

\section{Results}

Table 2 provides the results for the three different models. Model M1 resulted in a $p$ value higher than 0.05 for ethnicity after all non-significant interaction terms had been removed. Tested for a subgroup of 2,002 individuals, ethnicity therefore did not appear to affect the probability of having mature clavicles. Including this variable in the model does not improve the model fit. Exploring model M1 therefore effectively resulted in model M2, which could be fitted to the data of 3,256 individuals.

From model M2, it appeared that age, the HDI (i.e., socioeconomic status), sex, and the method of clavicle examination all significantly affected the probability of having completely fused clavicles. Exploring possible interaction between sex and age did not indicate a significant effect. The interaction term method $\times$ age, however, yielded a $p$ value of 0.000 . The subsequent assessment of the significance of the difference in $-2 \log$ likelihood between the resulting model and a nested model omitting the interaction term yielded a $p$ value of 0.000 . The Nagelkerke pseudo $R^{2}$ reduced from 0.815 to 0.795 after omission of the interaction term. All indicates a better model fit when the interaction term is included.

Model M3, which could be fitted to all available data on clavicle fusion, resulted in comparable model parameters for the dummy variable for CT scans. Confounding by an observer effect is therefore reduced.

\section{Application of the model}

The most appropriate model to predict the probability of being diagnosed with mature clavicles, i.e., providing the best model fit to the currently available data, appeared to be model M2. The resulting model-i.e., after the removal of non-significant main and interaction terms and using the parameters for covariates and the intercept - may be described by:

$$
\begin{aligned}
\ln [\mathrm{p} /(1-\mathrm{p})]= & -18.638+0.629^{\times} \text {age }+2.746^{\times} \mathrm{HDI} \\
& +\gamma_{\text {sex }}+\gamma_{\text {method }}+\beta_{\text {method }^{\times} \text {age }}{ }^{\times} \text {age. }
\end{aligned}
$$

Table 2 Estimated parameters of logistic regression models for the logit of the probability of mature clavicles

\footnotetext{
${ }^{a}$ The remaining parameters of model 1 (M1) are omitted from this section in the table, as final results for a model without the variable ethnicity are provided

\begin{tabular}{|c|c|c|c|c|}
\hline Model & Independent variables & $B$ & Standard error & $p$ value \\
\hline M1 $N=2,002$ & Ethnicity $^{\mathrm{a}}$ & & & 0.355 \\
\hline \multirow[t]{11}{*}{ M2 $N=3,256$} & Age & 0.629 & 0.034 & 0.000 \\
\hline & Sex (ref: male) & & & 0.026 \\
\hline & Female & 0.368 & 0.166 & \\
\hline & Human development index & 2.746 & 0.590 & 0.000 \\
\hline & Method (ref: dry bone specimen) & & & 0.000 \\
\hline & X-ray & -21.046 & 3.055 & \\
\hline & CT scan & -7.624 & 2.346 & \\
\hline & Method $\times$ age & & & 0.000 \\
\hline & X-ray & 1.029 & 0.135 & \\
\hline & CT scan & 0.423 & 0.104 & \\
\hline & Constant & -18.638 & 1.002 & 0.000 \\
\hline \multirow[t]{9}{*}{ M3 $N=3552$} & Age & 0.630 & 0.034 & 0.000 \\
\hline & Human development index & 3.012 & 0.584 & 0.000 \\
\hline & Method (ref: dry bone specimen) & & & 0.000 \\
\hline & $\mathrm{X}$-ray & -20.729 & 3.060 & \\
\hline & CT scan & -7.526 & 1.928 & \\
\hline & Method $\times$ age & & & 0.000 \\
\hline & X-ray & 1.023 & 0.135 & \\
\hline & CT scan & 0.401 & 0.083 & \\
\hline & Constant & -18.803 & 0.998 & 0.000 \\
\hline
\end{tabular}
for model 2 (M2).
} 
Parameters for the appropriate dummies for the categorical variables may be found in Table 2. From this equation, it follows that the predicted probability may be calculated using the following equation:

$$
\begin{aligned}
& \mathrm{p}(\text { age })=e^{\wedge}\left(\begin{array}{c}
-18.638+0.629^{\times} \text {age }+2.746^{\times} \mathrm{HDI} \\
+\gamma_{\text {sex }}+\gamma_{\text {method }}+\beta_{\text {method }^{\times} \text {age }} \times \text { age }
\end{array}\right) / \\
& \left(1+e^{\wedge} \cdot\left(\begin{array}{l}
-18.638+0.629^{\times} \text {age } \\
+2.746^{\times} \mathrm{HDI}+\gamma_{\mathrm{sex}}+\gamma_{\text {method }} \\
+\beta_{\text {method } \times \text { age }} \times \text { age }
\end{array}\right) .\right.
\end{aligned}
$$

Based on the currently available data on clavicle fusion, we can now predict the probability of having mature clavicles for different individuals. Here, we provide a few examples.

The predicted probability of mature clavicles is 0.016 (1.6\%) for a contemporary 19-year-old German male (HDI is 0.932 ). For a 20 - or 21-year-old German male, this probability increases to $0.029(2.9 \%)$ and $0.054(5.4 \%)$, respectively. For females, the predicted probability values are increased to $0.023(2.3 \%), 0.042(4.2 \%)$, and 0.076 (7.6\%), respectively. However, if the individuals have a lower socioeconomic status, these values are decreased. For contemporary 19-, 20-, and 21-year-old males from India (HDI is 0.611$)$, for instance, they are $0.007(0.7 \%), 0.012$ $(1.2 \%)$, and $0.023(2.3 \%)$, respectively. When diagnosed from X-ray or CT scan, the values increase. For the 21-yearold Indian male, it would be 0.040 (4\%) if diagnosed from an X-ray and $0.076(7.6 \%)$ if diagnosed from a CT scan (composed using a slice thickness that is far from optimal).

In Figs. 2 and 3, we show the differences in predicted probabilities at different ages between the various categories. In Fig. 2, we compare the predicted probability values for a German male with that for a German female and an Indian male. Figure 3 illustrates the difference in predicted probability for a German male depending on whether dry bone specimens, X-rays, or CT scans are examined.

\section{Discussion}

Ethnicity did not appear to significantly influence the probability of having completely fused clavicles, which is in line with the results of Schmeling et al. [12]. We should, however, keep in mind that the current database is not well balanced. In addition, a small number of individuals taken from McKern and Stewart [6] were misclassified in our analysis (see footnote Table 1). Additional data from future studies may contribute to the knowledge on the possible effect of ethnicity, if not strengthen our conclusion that there is no significant effect.

The effect of sex on the probability of having completely fused clavicles appeared significant. The estimated model parameter for the dummy variable for females being greater than zero indicates a positive effect on the logit of the probability. The predicted probability of mature clavicles is therefore greater for females than for males of similar ages. This would point to a generally earlier onset of fusion in females. A similar disparity has been reported for epiphysial closure of all other long bones [11]. There was no indication of significant interaction between sex and age. We may therefore assume similarly shaped probability curves for males and females, indicating an equal number of years during which complete fusion may generally be achieved.

A higher HDI, and therefore a higher socioeconomic status, appears to increase the probability of having mature clavicles. This would mean that under less preferable socioeconomic conditions, the process of fusion is generally delayed. This is in concordance with conclusions drawn by Schmeling et al. [12, 14], and was summarized before by Eveleth and Tanner [2].

The method of clavicle examination appeared to also be significant. The significant interaction term method $\times$ age indicates that we should assume a difference in the estimated effect of age depending on the method applied. For the age interval in which mature clavicles have been
Fig. 2 Predicted probability of having completely fused clavicles: a comparison between German males, German females and Indian males
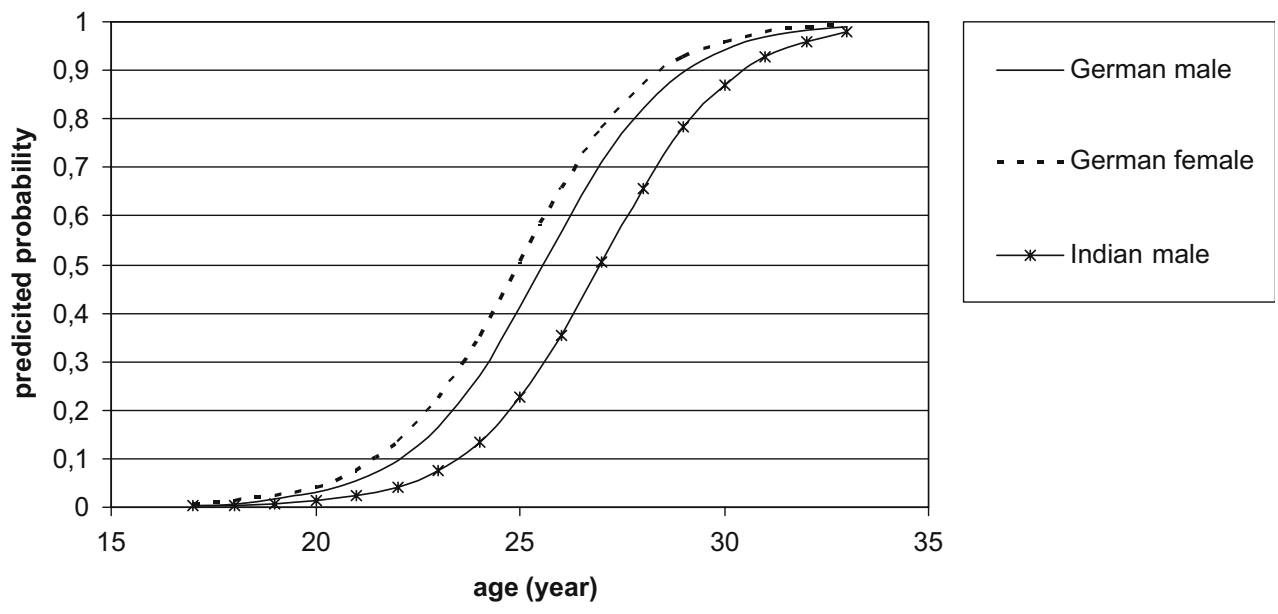
Fig. 3 Predicted probability of being diagnosed with mature clavicles: a comparison between examination by means of dry bone specimens, X-rays and CT scans
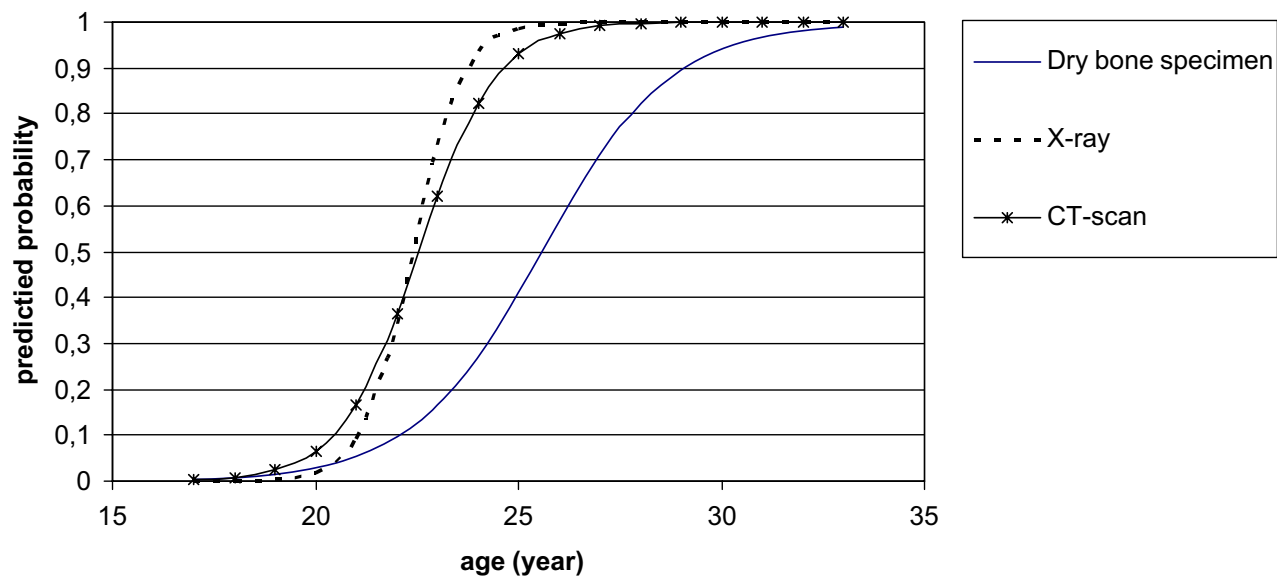

observed, the predicted probability of being diagnosed with mature clavicles is greater when X-rays or CT scans are used instead of dry bone specimens. It is noteworthy that the difference in the predicted probability is initially very small, but becomes increasingly greater with increasing age during a limited age interval. When keeping in mind that the curve for the predicted probability will be s-shaped, one may image that the difference is again only minimal for the older individuals.

A number of theories can be postulated to explain a difference in the predicted probabilities between methods of examination.

Inappropriate slice thickness Regarding the results from CT scans, one could postulate that the slice thickness affected the diagnosis. Mühler et al. [7] concluded that reconstructions composed with a slice thickness of $1 \mathrm{~mm}$ should be used. The vast majority of the analyzed data were, however, obtained from reconstructions with a greater slice thickness (up to $8 \mathrm{~mm}$ ) $[5,15]$. One can foresee that an almost completely fused clavicle may be diagnosed as being mature when details are lost due to the greater slice thickness. Schulze et al. [16] - from whom no data were included in our analysis, as no individual data were provided-observed two 19-year-olds with completely fused clavicles. Both observations were done from reconstructions with a slice thickness of $7 \mathrm{~mm}$ (Schulze, personal communication). We therefore think there is a realistic possibility of these cases having been misdiagnosed.

Persistent small grooves or notches between diaphysis and epiphysis When comparing the results from their study based on CT scans with two studies based on dry bone specimens [6, 9], Kreitner et al. [5] hypothesized that persistent small grooves or notches seen in dry bone specimens may be classified as partial fusions of the epiphysis with the clavicular shaft. This would reduce the probability of dry bone specimens being classified as completely fused.
Reduced visibility of the epiphyseal disc on X-rays X-rays used in the published studies have not been made with the purpose of examining the clavicles. Possibly, this has hindered a correct classification of the clavicle at times.

Interobserver variability As the sample included results from two studies based on X-rays and two studies based on CT scans, we hold it unlikely that what we perceive to be an effect of the applied method is in fact an observer effect.

Expectation bias In some X-rays, the (nearly fused) epiphyseal disc will have been more difficult to judge than in others. In such cases, knowing the actual age of the individuals could have affected the interpretation of the observation. Clavicles of relatively young individuals would then more often be classified as "not completely fused", while those of relatively old individuals would be classified as "completely fused" more often. We know, however, that in at least two out of four studies, one based on X-rays [13] and one on CT scans [15], the examiner was not aware of the age of the subjects when classifying the images. We therefore do not believe that expectation bias could have played a role of any great importance.

Statistical artifact In theory, it may be possible that due to an unfortunate coincidence, there were no relatively late maturing individuals examined by X-ray or CT scan.

In theory, all of these aspects could have contributed to the significant effect of the variable method. However, if significance of the interaction between method and age is not explained as a statistical artifact, only expectation bias would have resulted in a significant change in the shape of the probability curve. In case of misclassifications of nearly fused clavicles or differences in the classification system among studies, we would have expected a higher number of individuals with completely fused clavicles in the relatively young subjects, even below the age of 20 . 
If we, however, were to conclude that significance of the interaction term is in fact a statistical artifact, the term should be removed from the model, and model parameters should be recalculated. It is our ambition to investigate the matter further by comparing high quality CT scans, expected to allow a correct diagnosis, with X-rays taken from the same person.

\section{Conclusions}

The probability of having mature clavicles at a certain age is affected by the sex and socioeconomic status of the individual. The probability of being diagnosed with mature clavicles further appears affected by the method of clavicle examination. Predicted probabilities from the model may be used to illustrate acceptance or rejection of individual age claims.

Please note that the odds on having mature clavicles given a certain age should not be confused with the (posterior) odds of having reached a certain age given that the clavicles have matured. These probabilities are only equal if we assume a prior odds of 1 .

\section{References}

1. Black S, Scheuer L (1996) Age changes in the clavicle: from the early neonatal period to skeletal maturity. Int J Osteoarchaeol 6:425-434

2. Eveleth PB, Tanner JM (1991) Worldwide variation in human growth. Cambridge University Press, Cambridge

3. Ji L, Terazawa K, Tsukamoto T, Haga K (1994) Estimation of age from epiphyseal union degrees of the sternal end of the clavicle. Hokkaido Igaku Zasshi 69:104-111

4. Jit I, Kulkarni M (1976) Times of appearance and fusion of epiphysis at the medial end of the clavicle. Indian J Med Res 64:773-782

5. Kreitner KF, Schweden F, Riepert T, Nafe B, Thelen M (1998) Bone age determination based on the study of the medial extremity of the clavicle. Eur Radiol 8:1116-1122
6. McKern TW, Stewart TD (1957) Skeletal age changes in young American males analyzed from the standpoint of age identification. Technical Report EP 45, Quartermaster Research and Development Center, Environmental Protection Research Division. Natick, MA, pp 89-97

7. Mühler M, Schulz R, Schmidt S, Schmeling A, Reisinger W (2006) The influence of slice thickness on assessment of clavicle ossification in forensic age diagnostics. Int J Legal Med 120:15-17

8. Owings PA (1981) Epiphyseal union of the anterior iliac crest and medial clavicle in a modern multi-racial sample of males and females. MA thesis, California State University, Fullerton

9. Owings Webb PA, Meyers Suchey J (1985) Epiphyseal union of the anterior iliac crest and medial clavicle in a modern multi-racial sample of American males and females. Am J Phys Anthropol 68:457-466

10. Schaefer MC, Black SM (2005) Comparison of ages of epiphyseal union in North American and Bosnian skeletal material. J Forensic Sci 50:777-784

11. Scheuer L, Black S (2000) Developmental juvenile osteology. Academic, London

12. Schmeling A, Reisinger W, Loreck D, Vendura K, Markus W, Geserick G (2000) Effects of ethnicity on skeletal maturation: consequences for forensic age estimations. Int J Legal Med 113:253-258

13. Schmeling A, Schulz R, Reisinger W, Mühler M, Wernecke K, Geserick, G (2004) Studies on the time frame for ossification of the medial clavicular epiphyseal cartilage in conventional radiography. Int J Legal Med 118:5-8

14. Schmeling A, Schulz R, Danner B, Rosing FW (2006) The impact of economic progress and modernization in medicine on the ossification of hand and wrist. Int J Legal Med 120:121-126

15. Schulz R, Mühler M, Mutze S, Schmidt, S, Reisinger W, Schmeling A (2005) Studies on the time frame for ossification of the medial epiphysis of the clavicle as revealed by CT-scans. Int J Legal Med 119:142-145

16. Schulze D, Rother U, Fuhrmann A, Richel S, Faulmann G, Heiland M (2006) Correlation of age and ossification of the medial clavicular epiphysis using computed tomography. Forensic Sci Int 158:184-189

17. Stevenson PH (1924) Age order of epiphyseal union in man. Am J Phys Anthropol 7:53-93

18. Todd TW, D'Errico J (1928) The clavicular epiphyses. Am J Anat 4:25-50

19. Watkins K (2006) The 2006 human development report. Beyond scarcity: power, poverty and the global water crisis. United Nations Development Programme, New York 